\title{
Early steps towards quarks and their interactions using neutrino beams in CERN bubble chamber experiments
}

\author{
Don H. Perkins ${ }^{\mathrm{a}}$ \\ Department of Particle Physics and Astrophysics, University of Oxford, Denys Wilkinson \\ Building, Keble Rd, Oxford OX1 3RH, UK
}

\author{
Received 4 March 2016 / Received in final form 7 March 2016 \\ Published online 30 May 2016 \\ (c) The Author(s) 2016. This article is published with open access \\ at Springerlink.com
}

\begin{abstract}
Results from neutrino experiments at CERN in the1970's, using bubble chamber detectors filled with heavy liquids, gave early evidence for the existence of quarks and gluons as real dynamical objects. In detail, the measured moments of the non-singlet structure functions provided crucial support for the validity of the present theory of the strong inter-quark interactions, quantum chromodynamics.
\end{abstract}

\section{Early days}

The first dynamical evidence for point-like substructure in neutrons and protons was found in deep inelastic electron scattering experiments at Stanford, and was first presented by Panofsky at the IUPAP High Energy Physics Conference in Vienna in 1968. At that time, the results were described as evidence for "Bjorken scaling" or scale invariance in current algebra (Bjorken 1967), as indicated by a weak dependence of the structure functions describing deep inelastic scattering on $q^{2}$, the square of the 4-momentum transfer in the electron-nucleon collisions. This behaviour also suggested scattering from pointlike objects inside the nucleon, which Feynman (1969) labelled as partons, and which experiments were later to reveal as the long-sought quarks and gluons.

It is interesting to remark that tentative dynamical evidence for substructure in neutrons and protons had already appeared - but alas, was not recognised! - in the first neutrino experiment at CERN in 1963, in a small $1.2 \mathrm{~m}$ diameter heavy liquid bubble chamber (Block et al. 1964), at the same time as Gell-Mann and Zweig were inventing the quark model. After the 1968 presentation of deep inelastic electron scattering by nucleons, the observed linear energy dependence of the total neutrino cross-section (Budagov et al. 1969) was recognised as a crucial signal for point-like structure in the nucleon. Finally, definitive experiments with beams of both neutrinos and antineutrinos, to establish the precise nature of this substructure, in conjunction with the results on electron scattering, had to await the arrival in CERN of the much larger heavy liquid chamber Gargamelle in 1970.

a e-mail: d.perkins1@physics.ox.ac.uk 
In this report we recall some of the early results using neutrino and antineutrino beams from the CERN $25 \mathrm{GeV}$ PS accelerator, in the Gargamelle chamber with a freon $\left(\mathrm{CF}_{3} \mathrm{Br}\right.$ ) filling, in 1972/3; and those in 1978 from the CERN $200 \mathrm{GeV}$ SPS accelerator, in the BEBC chamber with a neon/hydrogen filling (Bosetti et al. 1982a). The neutrino beams, produced from decay in flight of pion and kaon secondaries created at the proton target, consisted principally of muon-type neutrinos (or antineutrinos), with weak interactions of the form $\nu_{\mu}+\mathrm{N} \rightarrow \mu^{-}+$hadrons. The BEBC chamber was equipped with an external muon identifier, which also enhanced the precision on the muon momentum measurements. The object of using heavy liquid fillings, with short nuclear interaction and radiation lengths, was to provide good detection efficiency for neutral hadrons and gamma rays, as well as large detecting mass.

The cross-sections were evaluated from the event rates and the neutrino/antineutrino fluxes, as determined from the muon fluxes measured at different depths in the shielding, and the $K / \pi$ ratios in front of the shielding (Bosetti et al. 1982b).

Together, these experiments were to provide early support for the quark model, with quarks and gluons detected as real dynamical objects, and for the asymptotically free gauge theory of the strong inter-quark interactions, quantum chromodynamics (QCD).

\section{Pointlike scattering}

For deep inelastic neutrino-nucleon charged current scattering using a muon-neutrino beam, the cross-section can be expressed in terms of the neutrino energy $E$, the muon energy $E_{\mu}$, the nucleon mass $M$, the four-momentum transfer squared, $q^{2}$, and the energy transfer to the nucleon, $\nu=E_{\text {hadron }}=E-E_{\mu}$. It has the form (neglecting the muon mass):

$$
\begin{aligned}
d^{2} \sigma^{\nu \mathrm{N}} / d x d y= & \left(G^{2} M E / \pi\right)\left[y^{2} x F_{1}^{\nu \mathrm{N}}\left(x, q^{2}\right)+(1-y-M x y / 2 E) F_{2}^{\nu \mathrm{N}}\left(x, q^{2}\right)\right. \\
& \left. \pm y(1-y / 2) x F_{3}^{\nu \mathrm{N}}\left(x, q^{2}\right)\right]
\end{aligned}
$$

where $F_{1}, F_{2}$ and $F_{3}$ are the so-called structure functions and the positive or negative sign in the last term applies for incident neutrinos or antineutrinos. Comparison of neutrino and antineutrino data and the observed $y$-dependence then allows evaluation of these three separate functions. The dimensionless "scaling variables" here are $x=$ $q^{2} / 2 M \nu$ and $y=\nu / E$, both varying over the range $0 \rightarrow 1$, while $G$ is the Fermi weak interaction constant. The above expression assumes an isoscalar $(N)$ target, with equal numbers of neutrons and protons, and that the weak interaction itself is pointlike (i.e. the mass of the mediating weak $W$ boson is very large). The third term, $M x y / 2 E$, in the bracket multiplying $F_{2}$ is very small at the energies considered here, and could be neglected. Small corrections, of order a few per cent, were applied to the observed cross-sections to take account of the fact that in freon $\left(\mathrm{CF}_{3} \mathrm{Br}\right)$ and in the neon-hydrogen mixtures employed, the $n / p$ ratio differed slightly from unity.

The corresponding expression for inelastic electron-nucleon scattering takes the form:

$$
d^{2} \sigma^{\mathrm{eN}} / d q^{2} d x=\left[4 \pi \alpha^{2} / q^{4}\right]\left\{(1-y) F_{2}^{\mathrm{eN}}\left(x, q^{2}\right) / x+\left(y^{2} / 2\right) 2 x F_{1}^{\mathrm{eN}}\left(x, q^{2}\right) / x\right\},
$$

where $F_{1}^{\mathrm{eN}}$ and $F_{2}^{\mathrm{eN}}$ are the electromagnetic structure functions (for magnetic and electric scattering respectively), analogous to the weak structure functions $F_{1}^{\nu \mathrm{N}}$ and $F_{2}^{\nu \mathrm{N}}$ in (1), and these can again be separated from the observed $y$-dependence. 
In the limit that $q^{2}$ becomes large, the ratio of structure functions,

$$
A=2 x F_{1}(x) / F_{2}(x) \rightarrow g / 2=1
$$

if the scattering takes place from pointlike constituents of half-integral spin and normal $(g=2)$ gyromagnetic ratio (Callan and Gross 1968). Both the electron and neutrino/antineutrino scattering results were in fair agreement with the Callan-Gross relation. Averaged over values of $x$ and $q^{2},\langle A\rangle=0.94 \pm 0.09$ was found in neutrino experiments (Bosetti et al. 1982a), while the electron scattering data gave $\langle A\rangle=0.94 \pm 0.04$ (Riordan et al. 1975). Similar results were found in muon scattering experiments at Fermilab (Anderson et al. 1976a, 1976b).

In the limit $y \rightarrow 0$, the cross-section (2) assumes the form

$$
d \sigma^{\mathrm{eN}} / d q^{2}=\left(4 \pi \alpha^{2} / q^{4}\right) \int F_{2}^{\mathrm{eN}}(x) d x / x
$$

so that comparing with the Rutherford scattering formula, the integral $\int F_{2}^{\mathrm{eN}}(x) d x / x$ is to be interpreted as a sum over all the parton constituents involved, weighted by the squares of their electrical charges.

Comparison of the results from electron and neutrino scattering led to the identification of the partons with the fractionally charged quarks proposed in the model of Gell-Mann and Zweig (1964). At the fairly low energies available in these early experiments, the quarks to be considered were the lightest quarks; the $d$ and $s$ quarks and antiquarks, with charges of $\pm e / 3$, and the $u$ quarks and antiquarks, with charges of $\pm 2 e / 3$. These were indeed the only "flavours" of quark known at that time. The heavier quarks $-c, b$ and $t$ - were to be discovered some time later, from 1974 onwards.

It should be remarked here that at this time, in the middle and late 1960's, extensive searches had been made in the strong interactions of meson and proton beams produced at accelerators, and in matter generally, aimed at the detection of the fractionally-charged quarks postulated by Gell-Mann and Zweig. These searches had all ended in failure. Not until much later was it realised that it was a peculiarity of the strong force itself that resulted in the phenomenon of "confinement". Thus quarks could not exist singly but only as components of the strongly-interacting hadrons. All attempts to "free" an individual quark would just result in more energy being stored in the gluon field, which is immediately converted into quark-antiquark pairs, that is to hadrons.

Nevertheless, the individual signals due to quarks could be detected due to the effect of their electrical and weak charges on bombarding beams of electrons, muons or neutrinos. The existence of that other component of the quark model, the neutral gluon and the carrier of the strong force, was first to be inferred from the results of the neutrino experiments and the conservation of energy and momentum.

The quantity $x$ in (3) is the fraction of the nucleon momentum carried by the quark or parton in a coordinate frame in which the nucleon has infinite momentum (Feynman 1969). Then the binding and transverse momentum components of the quark can be neglected, so that this is equivalent to having a hypothetical parton of mass $m$, free and stationary in the lab system. For such an elastic neutrino-parton collision, $q^{2}=2 m \nu$, where $\nu$ is the energy transfer, so that

$$
x=q^{2} / 2 M \nu=m / M
$$

is the fraction of the nucleon mass carried by the parton (in the infinite momentum frame).

Identifying the partons with the quarks and with their electric charges as indicated above, the value of $F_{2}$ on a proton target takes the form

$$
F_{2}^{e p}(x)=x\{(4 / 9)[u(x)+\bar{u}(x)]+(1 / 9)[d(x)+\bar{d}(x)+s(x) \bar{s}(x)]\},
$$


where for example $u(x)$ and $\bar{u}(x)$ represent the density of $u$ quarks and antiquarks with fraction $x$ of the proton momentum. On a neutron target, by isospin invariance, one simply interchanges the symbols $u$ and $d$. Hence on a nucleon target with equal numbers of neutrons and protons:

$$
F_{2}^{\mathrm{eN}}(x)=x\{(5 / 18)[u(x)+\bar{u}(x)+d(x)+\bar{d}(x)]+(1 / 9)[s(x)+\bar{s}(x)]\} .
$$

The corresponding structure function for the scattering of neutrinos and antineutrinos by point-like and stationary quark constituents of half-integral spin via the charged current weak interaction is simply

$$
F_{2}^{\nu \mathrm{N}}(x)=x\{u(x)+d(x)+\bar{u}(x)+\bar{d}(x)\}
$$

where the contribution of strange quarks is quite negligible, since it is suppressed by the factor $\sin ^{2} \theta_{c} \sim 0.04$ where $\theta_{c}$ is the Cabibbo angle.

From equations (6) and (7), one obtains the important relation

$$
F_{2}^{\nu \mathrm{N}}(x) \leqslant(18 / 5) F_{2}^{\mathrm{eN}}(x)=3.6 F_{2}^{\mathrm{eN}}(x),
$$

where the equality holds if one neglects the very small contribution from the "sea" of $s$ quark/antiquark pairs in electron scattering. Thus the value of $F_{2}$ measured in neutrino scattering is equal to that in electron scattering, multiplied by the reciprocal of the mean square charge of the effective quarks in the nucleon $\left(5 / 18\right.$ in units of $\left.e^{2}\right)$. The experimental value observed in 1972 for this ratio and reported at the IUPAP Chicago/Fermilab conferences was (Perkins 1972):

$$
\int F_{2}^{\nu \mathrm{N}}(x) d x / \int F_{2}^{\mathrm{eN}}(x) d x=3.4 \pm 0.7
$$

and was a decisive triumph for the quark model. Figure 1 shows early Gargamelle and SLAC data, comparing the two quantities in equation (8) as a function of $x$.

Uniquely, the neutrino/antineutrino data gave information on the antiquark content of the nucleon in high energy collisions, as well as on the number of valence quarks in the nucleon. The average value of the measured ratio $R \approx 0.50$ of antineutrino to neutrino total cross-sections yielded an estimate of $(3 R-1) /(3-R) \approx 0.2$ for the proportion of antiquarks in the nucleon, over the energy range considered.

The integrated value of the $F_{3}$ structure function, over the range $x=0 \rightarrow 1$, counts the number of valence quarks in the nucleon, that is $\int x F_{3}\left(x, q^{2}\right) d x / x \rightarrow 3$ in the limit $q^{2} \rightarrow \infty$ (Gross and Llewellyn-Smith 1969). The main experimental difficulty here was that since $F_{3}$ peaks near $x=0$, the contribution to the integral below $x_{\min }=q^{2} / 2 M E$ is missing. For $q^{2}=3-10 \mathrm{GeV}^{2}$, and $x_{\min }=0.02$, the integral had the value $2.7 \pm 0.4$, obviously compatible with the sumrule (Bosetti et al. 1978, see also Bolognese et al. 1983 for further analyses).

\section{Quark interactions (quantum chromodynamics, QCD)}

The gauge theory of quark interactions was proposed by Politzer (1974), Gross and Wilczek (1973, 1974) and Georgi and Politzer (1974). In this theory, the nucleon consists of three "valence" quarks, held together by exchange of vector gluons, plus a "sea" of quark-antiquark pairs. We now know that quarks can occur in 6 possible flavours - $u, d, s, c, b$ and $t$-although as explained above, at the energies encountered in these early experiments, only the lightest quarks $u, d$ and $s$ would be involved, and were indeed the only ones known at that time (pre-1974). 


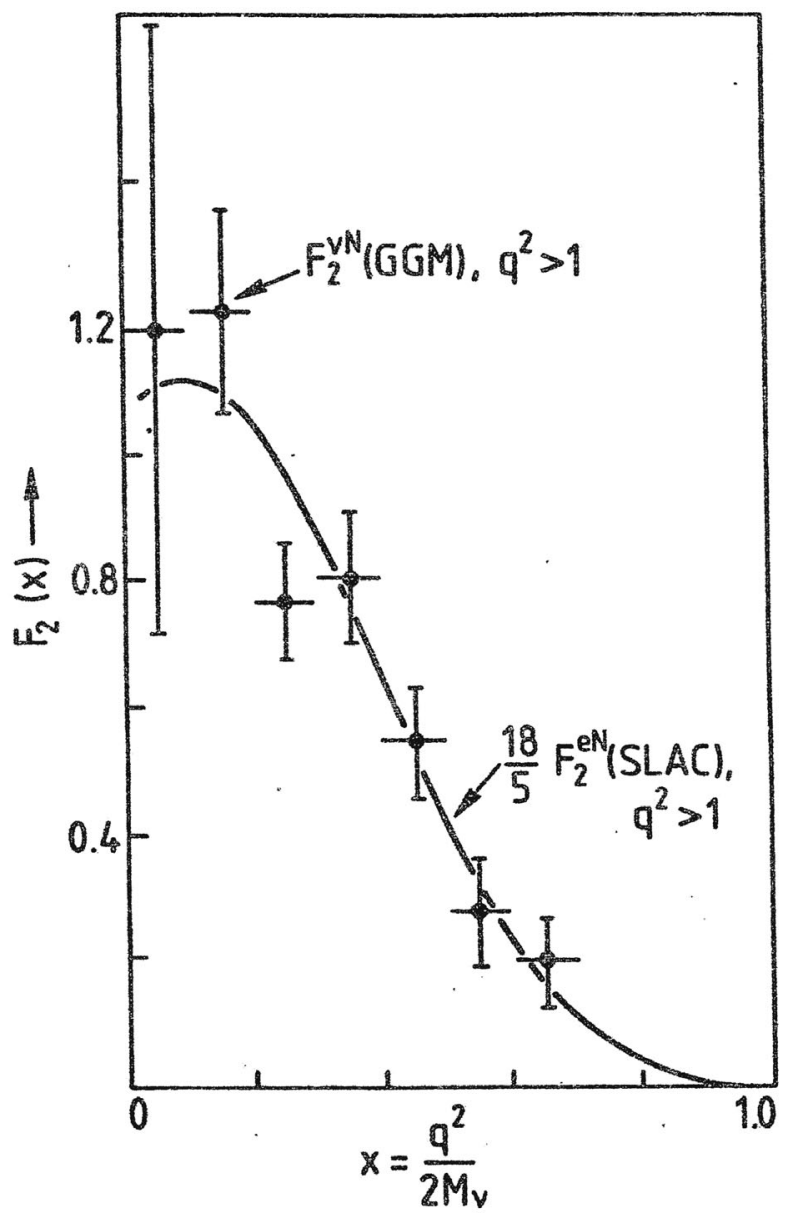

Fig. 1. The values of the weak and electromagnetic structure functions, $F_{2}^{\nu \mathrm{N}}(x)$ and $F_{2}^{\mathrm{eN}}(x)$ from the early neutrino, antineutrino and electron scattering experiments at CERN and SLAC. They are in good agreement when the electron data are divided by the mean square charge (5/18 in units of $\left.e^{2}\right)$ of the $u$ and $d$ quarks in the nucleon.

The integral of equation (7), that is $\int F_{2}^{\nu \mathrm{N}}(x) d x$, is a measure of the total fractional mass accounted for by the active quark and antiquark constituents in the nucleon, and reference to Figure 1 shows that this accounts for only about half of the nucleon mass. The rest $(0.50 \pm 0.05)$ is ascribed to the neutral gluon constituents mediating the quark-quark interactions.

In gauge theories, there is no obvious scale to the phenomena, so that a small element $d q^{2}$ will be proportional to $q^{2}$. The result is that, to first order, the strong quark-gluon coupling $\alpha_{s}$ depends logarithmically on $q^{2}$, as indicated in (14) below. One method employed to test the theory was by measurement of the moments of the structure functions. The non-singlet quark density $q_{\mathrm{NS}}\left(x, q^{2}\right)$ or $x F_{3}$ depends only on the valence quarks and is independent of the quark-antiquark "sea" contributions. The moments $M_{N S}$ of $q_{N S}\left(x, q^{2}\right)$ of order $N$ have the form, as shown by Altarelli and Parisi in 1977:

$$
d\left[M_{\mathrm{NS}}\left(N, q^{2}\right)\right] / d\left[\ln q^{2}\right]=\left[\alpha_{s}\left(q^{2}\right) / 2 \pi\right] A_{N} M_{\mathrm{NS}}\left(N, q^{2}\right),
$$


where

$$
M_{\mathrm{NS}}\left(N, q^{2}\right)=\int x^{\mathrm{N}-2} q_{\mathrm{NS}}\left(x, q^{2}\right) d x
$$

and

$$
A_{N}=\int z^{\mathrm{N}-2} P_{Q Q}(z) d z
$$

and the integrals are over the range $x, z=0 \rightarrow 1$. Here the "splitting function" $P_{\mathrm{QQ}}(x, y)$ for finding a quark with energy $x E$ originating from a quark of higher energy $y E$, which has radiated a gluon of energy $(y-x) E$ is, with $z=x / y$ :

$$
P_{\mathrm{QQ}}(z)=(4 / 3)\left(1+z^{2}\right) /(1-z) .
$$

In QCD the strong coupling in (10) has the value, to first order

$$
\alpha_{s}\left(q^{2}\right) / 2 \pi=6 /\left[(33-2 f)\left\{\ln q^{2} / \Lambda^{2}\right\}\right],
$$

where $f=3$ is the number of active quark flavours, and $\Lambda$ is a reference parameter which experimentally is known to be $\approx 0.2 \mathrm{GeV}$, as later observed in a variety of experiments over the $q^{2}$ range from 1 to $10^{4} \mathrm{GeV}^{2}$ (see Review of Particle Physics 2010). We note that, according to equation (14), the coupling becomes asymptotically free at large enough values of energy and $q^{2}$.

Integration of equation (10) yields the simple relation

$$
M_{\mathrm{NS}}=C_{N} /\left[\ln q^{2} / \Lambda^{2}\right]^{d(N)},
$$

where $C_{N}$ is a constant and $d(N)$ is the so-called anomalous dimension, with a value for the non-singlet component $\left(x F_{3}\right)$ of the structure function of

$$
d(N)=-6 A_{N} /(33-2 f)=[4 /(33-2 f)]\left[1-2 /\{N(N+1)\}+4 \sum_{2}^{N} 1 / j\right] .
$$

Thus if two different moments $N_{1}$ and $N_{2}$ are plotted against each other on a log scale, as $q^{2}$ varies, one should get a straight line with slope equal to the ratio of the anomalous dimensions, as shown in Figure 2, where the experimental points are compared with the slopes predicted by QCD. Note that in this comparison, the value of $\Lambda$ has dropped out. Results are shown from the BEBC and Gargamelle neutrino experiments (Bosetti et al. 1978) and also that from the CDHS counter experiment (de Groot et al. 1979a, 1979b), for the choices $N_{1}=4, N_{2}=6$; and $N_{1}=3, N_{2}=5$.

Equations (15) and (16) are valid for spin 1 (vector) gluons. For spin 0 (scalar) gluons, the final term in (16) has to be omitted, and the corresponding plots are shown by the dashed lines in Figure 2. It was clear from these plots that scalar gluons were excluded by the data.

In evaluating the moments, extra mass correction terms of the form $M^{2} / q^{2}$ can be significant at the energies involved, and these were taken into account using minor modifications to the scaling variables due to Nachtmann $(1973,1974)$. The corresponding modifications in evaluating the moments were given by Wandzura (1977).

Another possible source of correction which can enter the analysis can come from "high twist" terms, associated for example with quark transverse momentum effects, essentially arising from "cross talk" between the several quarks in the nucleon target. These effects are not directly calculable from theory but are expected to become important at low $q^{2}$ and high $x$ values. In fact, empirical formulae introducing higher twist terms did not markedly improve the fits to the data.

Finally, it is to be noted that the analysis described here was initially based on the assumption that the strong coupling $\alpha_{s} \ll 1$ at the energies involved. However when second-order $\left(\alpha_{s}^{2}\right)$ corrections were included in evaluating the moments, the effects on the predicted slopes were found to be only at the few per cent level (Bosetti et al. 1982a), and their inclusion did not alter the main features of the results or the conclusions drawn from them. 


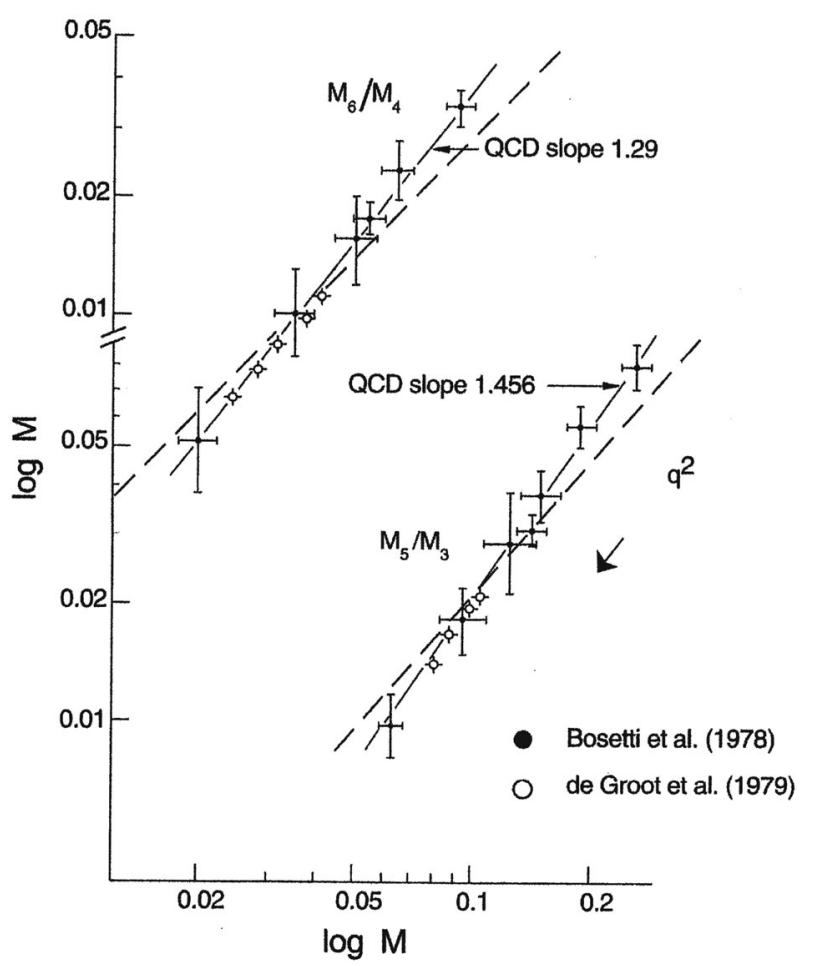

Fig. 2. Examples of log-log plots comparing ratios of different moments of the observed non-singlet (valence) quark distribution with first order predictions from QCD, shown by the solid lines, for vector gluons. The dashed lines show the prediction for scalar gluons. The values of $q^{2}$ range from $\sim 1 \mathrm{GeV}^{2}$ at top right to $\sim 100 \mathrm{GeV}^{2}$ at bottom left of these plots.

\section{Conclusions}

In summary, the main result of the above experiments and analysis, carried out over 35 years ago, was to provide, together with the electron scattering data, early support for the existence of quarks and gluons as real dynamical objects (rather than their original interpretation as the hypothetical generators of symmetry patterns found in the catalogue of baryon and meson bound states). These results also provided early and strong support for the validity of the current theory of the strong inter-quark interactions (quantum chromodynamics), with its property of asymptotic freedom. In particular, they gave this model much more credibility than other possible theories, for example fixed point theories of strong interactions. In such theories, the moments may also be related by power laws, but the values of the indices would be arbitrary, and not specified by the theory as in the case of QCD. Although later and more detailed analysis of more precise experiments have shown minor deviations from the linear plots shown in Figure 2, there is no question that these early experiments provided definitive support for the existence of quarks and gluons, as well as for the present theory of strong interactions.

Acknowledgements. In submitting this report, it is a pleasure to acknowledge the debt I owe to my colleague Dieter Haidt of the DESY laboratory, for his many proposals and suggestions at the various stages in its preparation. 


\section{References}

Altarelli G. and G. Parisi. 1977. Nucl. Phys. B 126: 298

Anderson H.L. et al. 1976. Phys. Rev. Lett. 36: 1422

Anderson H.L. et al. 1977. Phys. Rev. Lett. 38: 1450

Bjorken J. 1967. Phys. Rev. 163: 1767

Bloom E. et al. quoted by W.K.H. Panofsky at Vienna Conf. (1968)

Block M.M. et al. 1964. Phys. Lett. 12: 281

Bolognese T. et al. 1983. Phys. Rev. Lett. 50: 224

Bosetti P.C. et al. 1978. Nucl. Phys. B 142: 1

Bosetti P.C. et al. 1982a. Nucl. Phys. B 203: 362

Bosetti P.C. et al. 1982b. Phys. Lett. B 110: 167

Budagov I. et al. 1969. Phys. Lett. B 30: 364

Callan C.G. and D.G. Gross. 1968. Phys. Rev. Lett. 21: 311

Callan C.G. and D.G. Gross. 1969. Phys. Rev. Lett. 22: 156

de Groot J.G.H. et al. 1979a. Z. Phys. C 1: 143.

de Groot J.G.H. et al. 1979b. Phys. Lett. B 82: 292

Feynman R.P. 1969. Phys. Rev. Lett. 23: 1415

Gell-Mann M. 1964. Phys. Lett. 8: 214

Georgi H. and D. Politzer. 1974. Phys. Rev. D 9: 416

Gross D.J. and C.H. Llewellyn-Smith. 1969. Nucl. Phys. B 14: 337

Gross D.J. and F. Wilczek. 1973. Phys. Rev. D 8: 3633

Nachtmann O. 1973. Nucl. Phys. B 63: 237

Nachtmann O. 1974. Nucl. Phys. B 78: 455

Nakamura K. et al. 2010. J. Phys. G 37: 123

Perkins D.H. 1972. In IUPAP Conf. HEP, Chicago and Fermilab

Politzer D. 1974. Phys. Rep. C 14: 129

Riordan E.M. et al. SLAC-PUB 1634 (1975)

Wandzura S. 1977. Nucl. Phys. B 122: 412

Zweig G. 1964. CERN Report 8419/Th 412

Open Access This is an open access article distributed under the terms of the Creative Commons Attribution License (http://creativecommons.org/licenses/by/4.0), which permits unrestricted use, distribution, and reproduction in any medium, provided the original work is properly cited. 